\title{
Gas-phase metallicity of 27 galaxies at intermediate redshift ${ }^{\star} \star \star$ (Research Note)
}

\author{
L. Morelli ${ }^{1,2}$, V. Calvi ${ }^{1,3}$, A. Cardullo ${ }^{1}$, A. Pizzella ${ }^{1,2}$, E. M. Corsini ${ }^{1,2}$, and E. Dalla Bontà ${ }^{1,2}$ \\ 1 Dipartimento di Fisica e Astronomia "G. Galilei", Università di Padova, vicolo dell’Osservatorio 3, 35122 Padova, Italy \\ e-mail: lorenzo.morelli@unipd.it \\ 2 INAF - Osservatorio Astronomico di Padova, vicolo dell'Osservatorio 2, 35122 Padova, Italy \\ 3 Space Telescope Science Institute, 3700 San Martin Drive, Baltimore, MD 21218, USA \\ Received 30 March 2012 / Accepted 22 June 2012
}

\section{ABSTRACT}

\begin{abstract}
Aims. The purpose of this work is to make available new gas-phase oxygen abundance measurements for a serendipitous sample of 27 galaxies with redshifts $0.35 \leq z \leq 0.52$.

Methods. We measured the equivalent widths of the $\left[\mathrm{O}_{\mathrm{II}}\right] \lambda 3727, \mathrm{H} \beta$, and $\left[\mathrm{O}_{\mathrm{III}}\right] \lambda \lambda 4959,5007$ emission lines observed in the galaxy spectra obtained with the Visible Multi-Object Spectrograph mounted at the Very Large Telescope. For each galaxy, we derived the metallicity-sensitive emission-line ratio $R_{23}$, ionization-sensitive emission-line ratio $O_{32}$, and gas-phase oxygen abundance $12+\log (\mathrm{O} / \mathrm{H})$.

Results. The values of the gas-phase oxygen abundance $12+\log (\mathrm{O} / \mathrm{H})$ that we obtained for all the sample galaxies are consistent with previous findings for galaxies at intermediate redshift.
\end{abstract}

Key words. galaxies: distances and redshifts - galaxies: evolution - galaxies: formation - galaxies: fundamental parameters galaxies: ISM

\section{Introduction}

The gas-phase metallicity is one of the most important observational diagnostics of the current evolutionary state of galaxies. Accurate gas-phase metallicity measurements are crucial for investigating the interplay between fundamental processes, such as star formation, gas accretion, gas flows, and supernova-driven winds occurring during the life of galaxies (e.g., Moustakas et al. 2011; Cresci et al. 2012; Sommariva et al. 2012).

The galaxy gas-phase metallicity correlates with many properties, such as the star formation rate (Mannucci et al. 2010; Lara-López et al. 2010), morphological type (Edmunds \& Pagel 1984), surface mass density (Ryder 1995; Garnett et al. 1997), and maximum rotation velocity (Dalcanton 2007). In particular, the metallicity $Z$ most strongly correlates with the mass $M$ and $B$-band luminosity $L$ of the galaxy (McClure \& van den Bergh 1968; Skillman et al. 1989; Richer \& McCall 1995). Adopting the Sloan Digital Sky Survey (SDSS, Abazajian et al. 2009), Tremonti et al. (2004) and Gallazzi et al. (2005) derived the $M-Z$ and $L-Z$ relations in the local Universe for a large sample of galaxies on the basis of the gas-phase metallicity. These relations are very tight with a scatter of less than 0.1 dex and show that the more luminous galaxies have higher gas metallicities than their fainter counterparts, which has a great impact on the theoretical models of galaxy formation.

Another crucial piece of information to help us to understand the assembly history of galaxies comes from the study of the evolution of the $M-Z$ and $L-Z$ relations across cosmic time.

^ Based on observations made with ESO Telescopes at the La SillaParanal Observatory under programmes 075.B-0794 and 077.B-0767.

$\star \star$ Tables 1 and 3 are available in electronic form at http://www . aanda.org
This topic has been investigated by measuring the gas-phase metallicity of galaxies as a function of redshift up to $z \sim 3.5$. While these relations show clear evidence of galactic evolution at high $z$, because at a given mass higher- $z$ galaxies have lower metallicities (Shapley et al. 2004; Erb et al. 2006; Maiolino et al. 2008; Mannucci et al. 2009; Rodrigues et al. 2012), at intermediate redshift $(z<1)$ it is still debated whether there has been any evolution. Carollo \& Lilly (2001) and Kobulnicky \& Kewley (2004) found that the $L-Z$ relation at intermediate redshift is consistent with the local one. In contrast, Maier et al. (2005), Savaglio et al. (2005), and Zahid et al. (2011) measured lower metallicities for intermediate-redshift objects than for local galaxies, supporting a scenario in which the $L-Z$ and $M-Z$ relations also evolve over the range of redshifts between 0.5 and 1 .

In this research note, we therefore present new measurements of the equivalent widths for the $\left[\mathrm{O}_{\text {II }}\right] \lambda 3727, \mathrm{H} \beta$, [O III $] \lambda 4959$, and [O III] 25007 emission-lines and present our derived metallicity-sensitive emission lines ratios $R_{23}$, ionizationsensitive emission-lines ratios $O_{32}$, and gas-phase oxygen abundances $12+\log (\mathrm{O} / \mathrm{H})$ for 27 intermediate-redshift $(0.35 \leq z \leq$ $0.52)$ galaxies. The data that we analised for all the sample galaxies are available from the SDSS archive, hence our measurements are a valuable supplementary resource for the astronomical community.

\section{Observations and data reduction}

As often happens, the data acquired for a particular aim can potentially contain information useful for different purposes. For this work, we used observations carried out in 2006 at the Very Large Telescope (VLT) of the European Southern Observatory (ESO) at Paranal Observatory using the VIsible Multi-Object Spectrograph (VIMOS). The main goal of these observations 
was to find satellites in the region of $\sim 500 \times 500 \mathrm{kpc}^{2}$ surrounding seven nearby isolated spiral galaxies (Yegorova et al. 2011).

VIMOS was used in Multi Object Spectroscopy (MOS) mode with the grism "HR orange" and the order sortingfilter GG435. The grism is characterized by a reciprocal dispersion of $0.6 \AA$ pixel $^{-1}$ and a spectral resolution of $R=2150$ for the adopted 1.'0 slit. In the high-resolution configuration that we used for the observations, the spectral range strongly depends on the position of the slit in the field of view varying from $4550-6950 \AA$ to $6000-8400 \AA$. The full dataset includes MOS observations of 7 different fields for a total exposure time of $\sim 20 \mathrm{~h}$. This granted a typical exposure time of $\sim 3 \mathrm{~h}$ for each target field. Each of the four quadrants of VIMOS observed $\sim 50$ objects so as to have $\sim 200$ galaxies within each field of view of $16.0 \times 18.0$.

The observed sample consists of 1450 objects with SDSS $r$-band magnitudes ranging from $17 \mathrm{mag}$ to $24 \mathrm{mag}$ and a modal value of $22 \mathrm{mag}$. As expected, only a small fraction of the target galaxies were found to be satellites. We focused on the remaining 1347 background and foreground objects to measure their gas-phase metallicities. Their spectra were biassubtracted, corrected for flat-field effect, cleaned of cosmic rays, and wavelength-calibrated by means of the ESO VIMOS data reduction pipeline. A more detailed description of the data reduction is reported in Yegorova et al. (2011).

\section{Sample selection}

To estimate the gas-phase metallicity, it is necessary to know the electron temperature provided by the comparison between auroral and nebular emission lines (Osterbrock 1989). However, since auroral lines are generally weak, it is difficult to observe them in distant galaxies. To overcome this issue, empirical relations between the intensity of the nebular lines and metallicity have been used to derive the gas-phase metallicity. The most widely used metallicity indicator is the $R_{23}$ emission lines ratio parameter (Pagel et al. 1979) defined as

$R_{23}=\frac{[\mathrm{O} \text { II }] \lambda 3727+[\mathrm{O} \text { III }] \lambda 4959+[\mathrm{O} \text { III }] \lambda 5007}{\mathrm{H} \beta}$.

For our analysed dataset the signal-to-noise ratio $(S / N)$ of the spectra allowed us to measure redshifts for only 571 objects out of the observed 1347 galaxies. We fitted all the available emission lines with Gaussians. The [O II] $\lambda 3727$ doublet was modelled with two Gaussians of the same full width at half maximum. The galaxy redshift was computed as the average of the redshifts derived from the central wavelengths of the measured emission lines.

We detected 177 galaxies in the redshift range between 0.35 and 0.6. However, most of their spectra did not map all the $\left[\mathrm{O}_{\mathrm{II}}\right] \lambda 3727, \mathrm{H} \beta$, and $\left[\mathrm{O}_{\mathrm{III}}\right] \lambda \lambda 4959,5007$ emission-lines we needed to compute the metallicity-sensitive emission-lines ratio $R_{23}$, ionization-sensitive emission lines ratio $O_{32}$, and gas-phase oxygen abundance $12+\log (\mathrm{O} / \mathrm{H})$, because of their actual position in the VIMOS field of view. All the relevant emission lines fall in the observed spectral range only for 27 objects, which represent our final sample of galaxies. They are listed in Table 1 and the distribution of their measured redshifts is shown in Fig. 1.

\section{Data analysis and gas-phase metallicity}

The apparent $g$-band Petrosian magnitudes of the sample galaxies range between 24.9 and $20.8 \mathrm{mag}$, as they were derived from

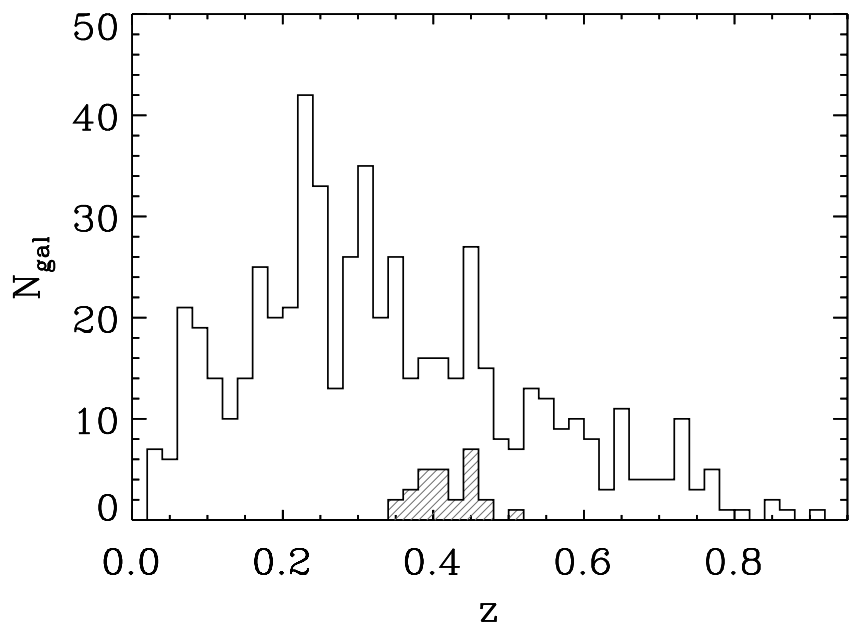

Fig. 1. Distribution of redshifts for the 571 galaxies with measured emission lines. The shaded histogram shows the distribution of the serendipitous sample of 27 objects presented in this work.

the SDSS DR7. Since the $S / N$ of the spectra was too low to derive the radial distribution of the gas metallicity, we rebinned the spectra along the spatial direction to obtain a $S / N \geq 15$ per resolution element.

Since no spectrophotometric standard star had been observed, the galaxy spectra were not flux-calibrated. Furthermore, the observed spectral range does not include the $\mathrm{H} \alpha$ emission line and thus a reliable reddening estimation was not possible. For the above reasons, we followed the approach suggested by Kobulnicky \& Phillips (2003) by replacing the flux of the emission-lines with their equivalent width when measuring the gas-phase metallicity. This method does not need flux-calibrated spectra and has the further advantage of being insensitive to reddening. In addition, Kobulnicky \& Phillips (2003) verified that the error associated with the use of the emission-line equivalent widths is smaller than both the typical error in the flux measurement and systematic error in the $R_{23}$ and $12+\log (\mathrm{O} / \mathrm{H})$ calibrations. Thus, we measured the equivalent widths of the $\left[\mathrm{O}_{\text {II }}\right] \lambda 3727, \mathrm{H} \beta$, and [O III] $] \lambda 44959,5007$ emission lines in therest frame spectra. For each emission line, we considered a central bandpass covering the feature of interest and two adjacent bandpasses, at the red and blue side, tracing the local continuum. The continuum level underlying the emission line was estimated by interpolating a straight line in the continuum bandpasses. The bandpasses were defined following Fisher et al. (1998) for [O II] $\lambda 3727$ and Gonzalez (1993) for $\mathrm{H} \beta$, [O III] $] \lambda 4959$, and [O III] $\lambda 5007$. The bandpasses are listed in Table 2 and shown in Fig. 2. The errors associated with the measured equivalent widths were derived from photon statistics and CCD read-out noise, and calibrated by means of Monte Carlo simulations.

A well-known problem in measuring the equivalent width of the $\mathrm{H} \beta$ emission line in galaxies is the contamination by the $\mathrm{H} \beta$ absorption line. We tried to address this issue by using GANDALF (Gas AND Absorption Line fitting; Sarzi et al. 2006) to fit the galaxy spectra with synthetic stellar population models, as done by Morelli et al. (2008, 2012). A linear combination of template stellar spectra from the ELODIE library by Prugniel \& Soubiran (2001) was convolved with the line-of-sight velocity distribution and fitted to the observed galaxy spectrum by $\chi^{2}$ minimization in pixel space. However, the low $S / N$ of the stellar continuum of the galaxy spectra prevented us from deriving a reliable combination of stellar templates to fit the galaxy 
Table 2. Definition of the bandpasses for the emission lines.

\begin{tabular}{lccc}
\hline \hline $\begin{array}{c}\text { Emission line } \\
(1)\end{array}$ & $\begin{array}{c}\text { Central bandpass } \\
{[\AA]}\end{array}$ & $\begin{array}{c}\text { Continuum bandpasses } \\
{[\AA]}\end{array}$ & Reference \\
& $(2)$ & $(3)$ & $(4)$ \\
\hline$\left[\mathrm{O}_{\text {II }}\right] \lambda 3727$ & $3716.30-3738.30$ & $3696.30-3716.30$ & 1 \\
& & $3738.30-3758.30$ & 1 \\
$\mathrm{H} \beta$ & $4851.32-4871.32$ & $4815.00-4845.00$ & 2 \\
& & $4880.00-4930.00$ & 2 \\
{$\left[\mathrm{O}_{\text {III }}\right] \lambda 4959$} & $4948.92-4968.92$ & $4885.00-4935.00$ & 2 \\
& & $5030.00-5070.00$ & 2 \\
{$\left[\mathrm{O}_{\text {III }}\right] \lambda 5007$} & $4996.85-5016.85$ & $4885.00-4935.00$ & 2 \\
& & $5030.00-5070.00$ & 2 \\
\hline
\end{tabular}

References. 1 = Fisher et al. (1998); 2 = Gonzalez (1993).

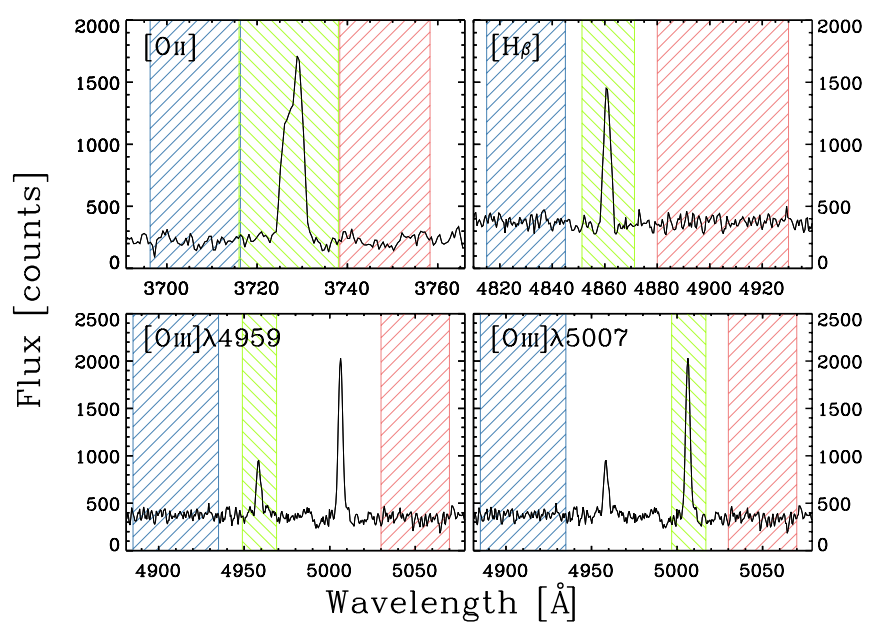

Fig. 2. Central (green dashed region) and continuum bandpasses (blue and red dashed regions) adopted for measuring the equivalent widths of the $\left[\mathrm{O}_{\mathrm{II}}\right] \lambda 3727$ (top left panel), $\mathrm{H} \beta$ (top right panel), [O III] $\lambda 4959$ (bottom left panel), and [O $\mathrm{III}] \lambda 5007$ (bottom right panel) emission lines.

stellar component. Therefore, to account for the $\mathrm{H} \beta$ absorptionline contamination we followed Kobulnicky \& Kewley (2004) by applying a correction of $2 \AA$ (Kobulnicky \& Phillips 2003) to the measured equivalent width of the $\mathrm{H} \beta$ emission line used to derive the gas-phase metallicity.

All the equivalent widths measured for the galaxies in our sample are reported in Table 1 . They were used to compute the metallicity-sensitive emission-line ratio $R_{23}$, as well as the emission-line ratio $O_{32}$ defined by Kobulnicky \& Kewley (2004) as

$O_{32}=\frac{[\mathrm{O} \text { III }] \lambda 4959+[\mathrm{O} \text { III }] \lambda 5007}{[\mathrm{O} \text { II }] \lambda 3727}$,

which is mostly sensitive to the ionization (Nagao et al. 2006). All the values of $\log \left(R_{23}\right)$ and $\log \left(O_{32}\right)$ are listed in Table 3.

For the sample galaxies, we could not derive the $\left[\mathrm{N}_{\mathrm{II}}\right] \lambda 6583 / \mathrm{H} \alpha$ ratio, thus it was impossible to break the $R_{23}$ degeneracy. Following Kobulnicky \& Zaritsky (1999) and Kobulnicky et al. (2003), we assumed that all the galaxies lie in the upper branch $(12+\log (\mathrm{O} / \mathrm{H}) \geq 8.4)$ of the $R_{23}-\mathrm{O} / \mathrm{H}$ relation. We then checked the consistency of this assumption by adopting the $\left[\mathrm{O}_{\mathrm{III}}\right] /\left[\mathrm{O}_{\mathrm{II}}\right]$ diagnostic lines ratio as proposed by Maiolino et al. (2008). As done by Kobulnicky \& Kewley (2004), we derived the gas-phase oxygen abundance by averaging the values obtained from the calibrations for the upper

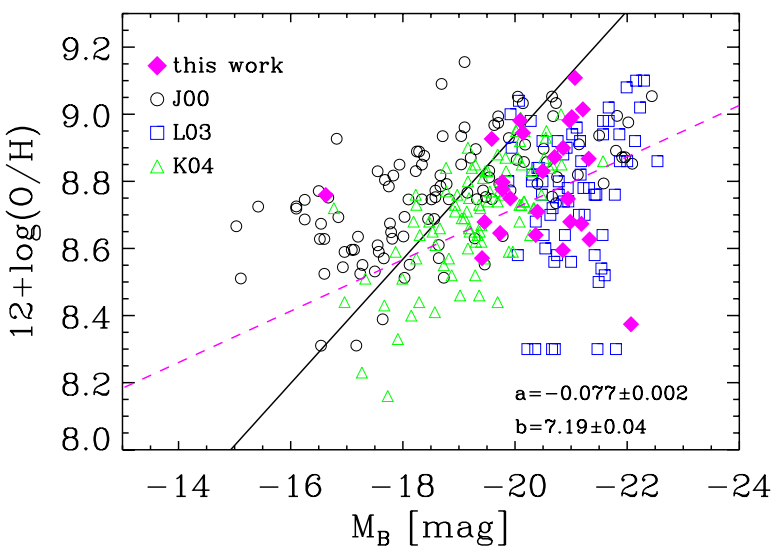

Fig. 3. Plot of our data (solid diamonds) on the $L-Z$ plane. Open squares represent the galaxies in the redshift range $0.47<z<0.92$ from the Canada-France Redshift Survey (Lilly et al. 2003), open triangles mark the galaxies with $0.3<z<0.6$ from the GOODS-North Field (Kobulnicky \& Kewley 2004), and open circles refer to the local galaxies of the Nearby Field Galaxy Sample (Jansen et al. 2000). The solid line indicates the $L-Z$ relation obtained by Tremonti et al. (2004) for local galaxies. The magenta dashed line represents the linear fit $\left(12+\log (\mathrm{O} / \mathrm{H})=\mathrm{a} M_{\mathrm{B}}+\mathrm{b}\right)$ through our data points except for $\mathrm{J} 154925.24-003717.8$ and J003806.59+000421.9.

branch given in McGaugh (1991) and Kewley \& Dopita (2002). The values of $12+\log (\mathrm{O} / \mathrm{H})$ and their uncertainties, owing to the statistical measurement errors in the equivalent widths, are listed in Table 3. The systematic errors due to the uncertainties in the photoionization models (0.2-0.5 dex; Kennicutt et al. 2003; Garnett et al. 2004) were not taken into account.

In Fig. 3, we plot the oxygen abundance as a function of the absolute magnitude in the $B$-band $M_{\mathrm{B}}$ for the sample galaxies. We derived $M_{\mathrm{B}}$ from the rest-frame $g$-band Petrosian magnitude given in the SDSS DR7, using the transformation $B=$ $g+0.327(g-r)+0.216$ (Chonis \& Gaskell 2008). The resulting values are given in Table 3 . We also plotted the data for local (Nearby Field Galaxy Sample, Jansen et al. 2000; SDSS, Tremonti et al. 2004) and intermediate-redshift galaxies (Canada-France Redshift Survey, Lilly et al. 2003; GOODSNorth Field, Kobulnicky \& Kewley 2004).

The dashed magenta line in Fig. 3 represents the linear fit to the sample galaxies. J154925.24-003717.8 and J003806.59+000421.9 are two outliers in the $L-Z$ distribution. The most prominent outlier is the faintest galaxy of the sample, for which the assumption of an upper $R_{23}-\mathrm{O} / \mathrm{H}$ branch could be incorrect (Skillman et al. 1989). Adopting the calibration of Kewley \& Dopita (2002) for the lower branch, we obtained a value of $12+\log (\mathrm{O} / \mathrm{H})=8.14$. However only the measurement of the $\left[\mathrm{N}_{\mathrm{II}}\right] \lambda 6583 / \mathrm{H} \alpha$ ratio could firmly help us to discriminate between the two branches. The second outlier has an extremely low value of the gas-phase metallicity. This is probably due to an underestimation of the $\mathrm{H} \beta$ absorption-line correction. The slope and zero-point of the fitted $L-Z$ relation to our data points (calculated after excluding the two outliers) are consistent within the errors with the slope and zero-point obtained by Kobulnicky \& Kewley (2004) for their sample of galaxies in the redshift range $0.2-0.4$. This was expected since 23 of our 27 galaxies have a redshift in the range $0.35-0.45$, and confirms their findings of an evolution in the $L-Z$ relation since intermediate redshift.

Finally, we estimated the star formation rate (SFR) of the sample galaxies from their $B$-band absolute magnitudes and $\mathrm{H} \beta$ equivalent widths according to the relations of 
Kennicutt et al. (2003) and Kobulnicky \& Kewley (2004)

$\operatorname{SFR}\left(M_{\odot} \mathrm{yr}^{-1}\right)=\frac{2.8 \times 5.49 \times 10^{31} \times 2.5^{M_{\mathrm{B}}} E W_{\mathrm{H} \beta}}{1.26 \times 10^{41}}$.

The derived SFRs are reported in Table 3.

\section{Conclusion}

Our original purpose was to explore the evolution of the $L-Z$ relation of Tremonti et al. (2004) to the intermediateredshift range using a large number of background galaxies in very deep VIMOS data. After combining all the constraints needed to perform our analysis, the number of objects dramatically decreased from 1347 to 27, preventing us from being able to draw any conclusion about the possible evolution of the $L-Z$ relation with redshift. Here, we have reported the measured equivalent widths of the $\left[\mathrm{O}_{\text {II }}\right] \lambda 3727$, $\mathrm{H} \beta$, [O III $] \lambda 4959$, and [O III] $\lambda 5007$ emission lines, the values of the metallicity-sensitive emission-line ratio $R_{23}$, the ionizationsensitive emission-line ratio $O_{32}$, and the gas-phase oxygen abundances $12+\log (\mathrm{O} / \mathrm{H})$ derived for the serendipitous sample of 27 galaxies with the aim of making them available to the community. Our measurements are consistent with those of Lilly et al. (2003) and Kobulnicky \& Kewley (2004) for galaxies in the redshift range $0.3<z<1.0$.

Acknowledgements. We thank the anonymous referee for comments that improved the completeness of this paper. This work was supported by Padua University through the grants CPDA089220/08, 60A02-5934/09, and 60A02$1283 / 10$ and by the Italian Space Agency through the grant ASI-INAF I/009/10/0. L.M. acknowledges financial support from Padua University grant CPS0204. V. C. is grateful to STScI for hospitality during the preparation of this paper.

\section{References}

Abazajian, K. N., Adelman-McCarthy, J. K., Agüeros, M. A., et al. 2009, ApJS, 182,543

Carollo, C. M., \& Lilly, S. J. 2001, ApJ, 548, L153

Chonis, T. S., \& Gaskell, C. M. 2008, AJ, 135, 264

Cresci, G., Mannucci, F., Sommariva, V., et al. 2012, MNRAS, 421, 262

Dalcanton, J. J. 2007, ApJ, 658, 941
Edmunds, M. G., \& Pagel, B. E. J. 1984, MNRAS, 211, 507

Erb, D. K., Shapley, A. E., Pettini, M., et al. 2006, ApJ, 644, 813

Fisher, D., Fabricant, D., Franx, M., \& van Dokkum, P. 1998, ApJ, 498, 195

Gallazzi, A., Charlot, S., Brinchmann, J., White, S. D. M., \& Tremonti, C. A. 2005, MNRAS, 362, 41

Garnett, D. R., Shields, G. A., Skillman, E. D., Sagan, S. P., \& Dufour, R. J. 1997, ApJ, 489, 63

Garnett, D. R., Kennicutt, Jr., R. C., \& Bresolin, F. 2004, ApJ, 607, L21

Gonzalez, J. J. 1993, Ph.D. Thesis, Univ. California, Santa Cruz

Jansen, R. A., Fabricant, D., Franx, M., \& Caldwell, N. 2000, ApJS, 126, 331

Kennicutt, Jr., R. C., Bresolin, F., \& Garnett, D. R. 2003, ApJ, 591, 801

Kewley, L. J., \& Dopita, M. A. 2002, ApJS, 142, 35

Kobulnicky, H. A., \& Kewley, L. J. 2004, ApJ, 617, 240

Kobulnicky, H. A., \& Phillips, A. C. 2003, ApJ, 599, 1031

Kobulnicky, H. A., \& Zaritsky, D. 1999, ApJ, 511, 118

Kobulnicky, H. A., Willmer, C. N. A., Phillips, A. C., et al. 2003, ApJ, 599, 1006

Lara-López, M. A., Bongiovanni, A., Cepa, J., et al. 2010, A\&A, 519, A31

Lilly, S. J., Carollo, C. M., \& Stockton, A. N. 2003, ApJ, 597, 730

Maier, C., Lilly, S. J., Carollo, C. M., Stockton, A., \& Brodwin, M. 2005, ApJ, 634,849

Maiolino, R., Nagao, T., Grazian, A., et al. 2008, A\&A, 488, 463

Mannucci, F., Cresci, G., Maiolino, R., et al. 2009, MNRAS, 398, 1915

Mannucci, F., Cresci, G., Maiolino, R., Marconi, A., \& Gnerucci, A. 2010, MNRAS, 408, 2115

McClure, R. D., \& van den Bergh, S. 1968, AJ, 73, 1008

McGaugh, S. S. 1991, ApJ, 380, 140

Morelli, L., Pompei, E., Pizzella, A., et al. 2008, MNRAS, 389, 341

Morelli, L., Corsini, E. M., Pizzella, A., et al. 2012, MNRAS, 423, 962

Moustakas, J., Zaritsky, D., Brown, M., et al. 2011, ApJ, submitted [arXiv: 1112.3300]

Nagao, T., Maiolino, R., \& Marconi, A. 2006, A\&A, 459, 85

Osterbrock. 1989, Astrophysics of Gaseous Nebulae and Active Galactic Nuclei (Mill Valley: University Science Books)

Pagel, B. E. J., Edmunds, M. G., Blackwell, D. E., Chun, M. S., \& Smith, G. 1979, MNRAS, 189, 95

Prugniel, P., \& Soubiran, C. 2001, A\&A, 369, 1048

Richer, M. G., \& McCall, M. L. 1995, ApJ, 445, 642

Rodrigues, M., Puech, M., Hammer, F., Rothberg, B., \& Flores, H. 2012, MNRAS, 421, 2888

Ryder, S. D. 1995, ApJ, 444, 610

Sarzi, M., Falcón-Barroso, J., Davies, R. L., et al. 2006, MNRAS, 366, 1151

Savaglio, S., Glazebrook, K., Le Borgne, D., et al. 2005, ApJ, 635, 260

Shapley, A. E., Erb, D. K., Pettini, M., Steidel, C. C., \& Adelberger, K. L. 2004, ApJ, 612, 108

Skillman, E. D., Kennicutt, R. C., \& Hodge, P. W. 1989, ApJ, 347, 875

Sommariva, V., Mannucci, F., Cresci, G., et al. 2012, A\&A, 539, A136

Tremonti, C. A., Heckman, T. M., Kauffmann, G., et al. 2004, ApJ, 613, 898

Yegorova, I. A., Pizzella, A., \& Salucci, P. 2011, A\&A, 532, A105

Zahid, H. J., Kewley, L. J., \& Bresolin, F. 2011, ApJ, 730, 137 
Table 1. Measured properties of the sample galaxies.

\begin{tabular}{|c|c|c|c|c|c|}
\hline $\begin{array}{l}\text { Galaxy name } \\
\text { SDSS } \\
\text { (1) }\end{array}$ & $\begin{array}{c}{[\mathrm{O} \text { пा] }] 33727} \\
{[\AA]} \\
(2)\end{array}$ & $\begin{array}{l}\mathrm{H} \beta \\
{[\AA \AA]} \\
(3)\end{array}$ & $\begin{array}{c}{[\mathrm{O} \text { III] }] \lambda 4959} \\
{[\AA]} \\
(4)\end{array}$ & $\begin{array}{c}{[\mathrm{O} \text { III] }] \lambda 5007} \\
{[\AA]} \\
(5)\end{array}$ & (6) \\
\hline J003845.31+000006.8 & $2.95 \pm 0.45$ & $1.43 \pm 0.49$ & $10.01 \pm 0.66$ & $26.35 \pm 0.45$ & 0.451 \\
\hline J003838.68+000225.1 & $92.41 \pm 0.68$ & $22.22 \pm 0.31$ & $15.58 \pm 0.72$ & $46.25 \pm 0.69$ & 0.408 \\
\hline J003806.59+000421.9 & $208.77 \pm 7.16$ & $15.75 \pm 1.07$ & $9.98 \pm 2.50$ & $39.45 \pm 1.30$ & 0.474 \\
\hline $\mathrm{J} 134235.94+014424.1$ & $3.89 \pm 0.41$ & $3.78 \pm 0.54$ & $1.61 \pm 0.46$ & $9.17 \pm 0.68$ & 0.411 \\
\hline $\mathrm{J} 134229.37+015244.5$ & $58.21 \pm 0.58$ & $20.63 \pm 0.48$ & $17.33 \pm 0.77$ & $43.93 \pm 0.69$ & 0.372 \\
\hline J134156.84+014241.0 & $6.09 \pm 0.47$ & $4.79 \pm 1.57$ & $3.74 \pm 0.87$ & $13.11 \pm 0.82$ & 0.390 \\
\hline $\mathrm{J} 145221.51+043448.8$ & $23.01 \pm 0.55$ & $9.14 \pm 0.15$ & $-0.12 \pm 0.45$ & $5.17 \pm 0.35$ & 0.450 \\
\hline J145150.75+044111.6 & $7.09 \pm 0.37$ & $3.23 \pm 0.83$ & $2.74 \pm 0.78$ & $2.76 \pm 0.40$ & 0.425 \\
\hline $\mathrm{J} 145147.24+043654.2$ & $30.29 \pm 1.13$ & $6.28 \pm 1.68$ & $4.61 \pm 1.35$ & $6.12 \pm 1.17$ & 0.517 \\
\hline J145156.20+043321.7 & $7.92 \pm 0.61$ & $2.16 \pm 0.12$ & $4.13 \pm 0.25$ & $14.93 \pm 0.32$ & 0.416 \\
\hline $\mathrm{J} 145147.77+043244.5$ & $11.84 \pm 0.61$ & $1.03 \pm 0.79$ & $2.70 \pm 0.75$ & $4.18 \pm 0.81$ & 0.414 \\
\hline $\mathrm{J} 145149.20+043501.6$ & $14.03 \pm 1.09$ & $2.90 \pm 0.50$ & $1.57 \pm 0.84$ & $8.16 \pm 0.67$ & 0.443 \\
\hline $\mathrm{J} 152638.18+034353.8$ & $15.42 \pm 0.36$ & $9.75 \pm 0.46$ & $4.80 \pm 0.79$ & $15.49 \pm 0.51$ & 0.407 \\
\hline $\mathrm{J} 152630.18+035110.8$ & $19.58 \pm 0.57$ & $5.93 \pm 0.45$ & $3.27 \pm 1.01$ & $4.39 \pm 0.60$ & 0.458 \\
\hline J152559.83+035249.3 & $18.67 \pm 0.45$ & $3.24 \pm 0.59$ & $3.30 \pm 0.67$ & $8.50 \pm 0.56$ & 0.475 \\
\hline $\mathrm{J} 152606.03+034530.7$ & $34.36 \pm 0.54$ & $9.08 \pm 0.65$ & $6.12 \pm 0.44$ & $30.29 \pm 0.36$ & 0.386 \\
\hline J154933.50-004255.0 & $39.71 \pm 0.57$ & $14.98 \pm 0.27$ & $14.95 \pm 0.68$ & $51.32 \pm 0.46$ & 0.358 \\
\hline J154931.48-003406.1 & $45.92 \pm 0.59$ & $21.36 \pm 0.62$ & $28.59 \pm 0.42$ & $107.08 \pm 0.36$ & 0.386 \\
\hline J154925.24-003717.8 & $18.21 \pm 0.45$ & $4.76 \pm 0.63$ & $2.99 \pm 0.32$ & $13.41 \pm 0.32$ & 0.386 \\
\hline J154914.49-004010.2 & $30.77 \pm 0.65$ & $9.05 \pm 0.43$ & $4.52 \pm 0.89$ & $11.21 \pm 0.51$ & 0.441 \\
\hline J154925.12-003645.4 & $18.54 \pm 0.34$ & $5.50 \pm 0.45$ & $1.90 \pm 0.19$ & $7.83 \pm 0.37$ & 0.445 \\
\hline J222013.98-074355.8 & $6.41 \pm 0.47$ & $2.06 \pm 0.20$ & $1.00 \pm 0.32$ & $1.29 \pm 0.24$ & 0.399 \\
\hline J222014.86-073333.7 & $23.12 \pm 1.01$ & $7.10 \pm 0.88$ & $1.51 \pm 1.52$ & $10.07 \pm 1.57$ & 0.440 \\
\hline J221934.90-073825.9 & $7.73 \pm 0.89$ & $3.44 \pm 0.29$ & $8.27 \pm 0.42$ & $26.36 \pm 0.35$ & 0.433 \\
\hline J221941.85-074703.8 & $14.91 \pm 0.58$ & $2.52 \pm 0.57$ & $2.88 \pm 0.52$ & $2.38 \pm 0.34$ & 0.369 \\
\hline J221939.28-074744.5 & $1.72 \pm 0.37$ & $0.88 \pm 0.14$ & $0.31 \pm 0.34$ & $0.62 \pm 0.32$ & 0.351 \\
\hline J221941.90-074825.7 & $29.10 \pm 0.60$ & $7.12 \pm 0.49$ & $6.26 \pm 0.63$ & $15.38 \pm 0.21$ & 0.375 \\
\hline
\end{tabular}

Notes. Column (1): SDSS name of the galaxy. Columns (2-5): equivalent widths of the $\left[\mathrm{O}_{\mathrm{II}}\right] \lambda 3727, \mathrm{H} \beta$, [O III $] \lambda 4959$, and $\left[\mathrm{O}_{\mathrm{III}}\right] \lambda 5007$ emission lines, respectively. Column (6): measured redshift. 
Table 3. Derived properties of the sample galaxies.

\begin{tabular}{|c|c|c|c|c|c|}
\hline $\begin{array}{l}\text { Galaxy name } \\
\text { SDSS } \\
(1)\end{array}$ & $\begin{array}{c}M_{\mathrm{B}} \\
{[\mathrm{mag}]} \\
(2) \\
\end{array}$ & $\begin{array}{c}\log \left(R_{23}\right) \\
(3)\end{array}$ & $\log \left(O_{32}\right)$ & $\begin{array}{c}12+\log (\mathrm{O} / \mathrm{H}) \\
(5)\end{array}$ & $\begin{array}{c}\mathrm{SFR} \\
{\left[M_{\odot} \mathrm{yr}^{-1}\right]} \\
(6)\end{array}$ \\
\hline J003845.31+000006.8 & -22.06 & $1.06_{-0.01}^{+0.01}$ & $1.09_{-0.07}^{+0.06}$ & $8.37 \pm 0.03$ & 1.05 \\
\hline $\mathrm{J} 003838.68+000225.1$ & -21.32 & $0.80_{-0.02}^{+0.01}$ & $-0.17_{-0.02}^{+0.01}$ & $8.63 \pm 0.02$ & 8.33 \\
\hline J003806.59+000421.9 & -21.46 & $1.16_{-0.01}^{-0.02}$ & $-0.62_{-0.03}^{-0.02}$ & $7.72 \pm 0.06$ & 6.71 \\
\hline $\mathrm{J} 134235.94+014424.1$ & -20.09 & $0.40_{-0.02}^{+0.01}$ & $0.44_{-0.06}^{+0.05}$ & $8.98 \pm 0.02$ & 0.45 \\
\hline $\mathrm{J} 134229.37+015244.5$ & -19.91 & $0.72_{-0.01}^{+0.01}$ & $0.02_{-0.03}^{+0.00}$ & $8.75 \pm 0.01$ & 2.11 \\
\hline $\mathrm{J} 134156.84+014241.0$ & -19.58 & $0.53_{-0.02}^{+0.01}$ & $0.44_{-0.04}^{+0.03}$ & $8.93 \pm 0.02$ & 0.36 \\
\hline $\mathrm{J} 145221.51+043448.8$ & -20.97 & $0.40_{-0.01}^{+0.02}$ & $-0.65_{-0.05}^{+0.04}$ & $8.98 \pm 0.03$ & 2.46 \\
\hline $\mathrm{J} 145150.75+044111.6$ & -21.01 & $0.38_{-0.03}^{+0.01}$ & $-0.10_{-0.08}^{+0.06}$ & $8.99 \pm 0.04$ & 0.90 \\
\hline $\mathrm{J} 145147.24+043654.2$ & -20.94 & $0.69_{-0.02}^{+0.02}$ & $-0.45_{-0.08}^{+0.06}$ & $8.75 \pm 0.06$ & 1.65 \\
\hline $\mathrm{J} 145156.20+043321.7$ & -21.18 & $0.81_{-0.01}^{+0.01}$ & $0.38_{-0.03}^{+0.03}$ & $8.67 \pm 0.02$ & 0.70 \\
\hline $\mathrm{J} 145147.77+043244.5$ & -20.36 & $0.79_{-0.03}^{+0.01}$ & $-0.23_{-0.08}^{+0.06}$ & $8.64 \pm 0.07$ & 0.16 \\
\hline $\mathrm{J} 145149.20+043501.6$ & -19.77 & $0.68_{-0.02}^{+0.03}$ & $-0.15_{-0.06}^{+0.05}$ & $8.78 \pm 0.05$ & 0.26 \\
\hline $\mathrm{J} 152638.18+034353.8$ & -20.14 & $0.48_{-0.01}^{+0.02}$ & $0.11_{-0.02}^{+0.02}$ & $8.94 \pm 0.01$ & 1.23 \\
\hline $\mathrm{J} 152630.18+035110.8$ & -20.85 & $0.53_{-0.02}^{+0.01}$ & $-0.40_{-0.07}^{+0.06}$ & $8.90 \pm 0.04$ & 1.44 \\
\hline $\mathrm{J} 152559.83+035249.3$ & -20.98 & $0.76_{-0.01}^{-0.02}$ & $-0.19_{-0.03}^{+0.03}$ & $8.68 \pm 0.03$ & 0.88 \\
\hline $\mathrm{J} 152606.03+034530.7$ & -19.73 & $0.80_{-0.02}^{+0.01}$ & $0.02_{-0.01}^{+0.04}$ & $8.64 \pm 0.01$ & 0.78 \\
\hline J154933.50-004255.0 & -19.45 & $0.79_{-0.02}^{+0.02}$ & $0.22_{-0.01}^{+0.01}$ & $8.68 \pm 0.02$ & 1.00 \\
\hline J154931.48-003406.1 & -19.41 & $0.89_{-0.03}^{+0.02}$ & $0.47_{-0.02}^{-0.02}$ & $8.57 \pm 0.03$ & 1.38 \\
\hline J154925.24-003717.8 & -16.62 & $0.71_{-0.01}^{+0.03}$ & $-0.04_{-0.01}^{+0.02}$ & $8.76 \pm 0.01$ & 0.02 \\
\hline $\mathrm{J} 154914.49-004010.2$ & -20.49 & $0.62_{-0.01}^{+0.01}$ & $-0.29_{-0.03}^{+0.01}$ & $8.83 \pm 0.02$ & 1.57 \\
\hline J154925.12-003645.4 & -20.70 & $0.58_{-0.01}^{+0.01}$ & $-0.28_{-0.02}^{+0.02}$ & $8.87 \pm 0.01$ & 1.16 \\
\hline J222013.98-074355.8 & -21.21 & $0.33_{-0.03}^{+0.01}$ & $-0.44_{-0.09}^{+0.02}$ & $9.01 \pm 0.05$ & 0.69 \\
\hline J222014.86-073333.7 & -21.31 & $0.58_{-0.03}^{+0.03}$ & $\begin{array}{r}-0.09 \\
-0.30_{-0.09}^{+0.07}\end{array}$ & $8.87 \pm 0.06$ & 2.62 \\
\hline J221934.90-073825.9 & -20.85 & $0.89_{-0.01}^{+0.03}$ & $0.65_{-0.05}^{+0.04}$ & $8.59 \pm 0.02$ & 0.83 \\
\hline J221941.85-074703.8 & -19.77 & $0.65_{-0.01}^{+0.01}$ & $-0.45_{-0.05}^{+0.05}$ & $8.80 \pm 0.04$ & 0.22 \\
\hline J221939.28-074744.5 & -21.06 & $0.04_{-0.09}^{-0.01}$ & $-0.26_{-0.034}^{-0.05}$ & $9.11 \pm 0.13$ & 0.25 \\
\hline J221941.90-074825.7 & -20.39 & $0.74_{-0.01}^{+0.01}$ & $-0.12_{-0.01}^{+0.04}$ & $8.71 \pm 0.01$ & 1.13 \\
\hline
\end{tabular}

Notes. Column (2): absolute $B$-band magnitude from SDSS DR7 derived adopting the measured redshift. Column (3): metallicity-sensitive $R_{23}$ parameter. Column (4): ionization-sensitive $O_{32}$ parameter. Column (5): Gas-phase oxygen abundance. Column (6): star formation rate as derived using the $\mathrm{H} \beta$ uncorrected for $\mathrm{H} \beta$ stellar absorption line. 